\title{
Role of routine computed tomography in paediatric pleural empyema
}

\author{
A Jaffe, ${ }^{1,2,3}$ A D Calder, ${ }^{4}$ C M Owens, ${ }^{4}$ S Stanojevic, ${ }^{2}$ S Sonnappa ${ }^{1,2}$
}

- Additional Methods data are published online only at http:// thorax.bmj.com/content/vol63/ issue 10

${ }^{1}$ Department of Respiratory Medicine, Great Ormond Street Hospital for Children NHS Trust, London, UK; ${ }^{2}$ Portex

Anaesthesia, Intensive Therapy and Respiratory Unit, Institute of Child Health, London, UK:

${ }^{3}$ Sydney Children's Hospital, Randwick and University of New South Wales, Sydney, Australia;

${ }^{4}$ Department of Radiology, Great Ormond Street Hospital for Children NHS Trust, London, UK

Correspondence to:

Dr S Sonnappa, Portex

Anaesthesia, Intensive Therapy and Respiratory Unit, Level 6,

Cardiac Wing, Institute of Child

Health, 30 Guilford Street,

London WC1N 1EH, UK:

s.sonnappa@ich.ucl.ac.uk

Received 2 December 2007

Accepted 29 April 2008

Published Online First

20 May 2008

\section{ABSTRACT}

Background: The incidence of empyema in children is increasing worldwide. While there are emerging data for the best treatment options, there is little evidence to support the imaging modalities used to guide treatment, particularly with regard to the role of routine CT scanning. The aims of this study were to develop a radiological scoring system for paediatric empyema and to assess the utility of routine CT scanning in this disease.

Methods: Children with empyema were prospectively enrolled over a 3-year period into a randomised clinical trial of video-assisted thoracoscopic surgery versus percutaneous chest drain insertion and urokinase. All children received a preoperative chest radiograph (CXR), pleural ultrasound scan (USS) and chest CT scan. In the urokinase arm the clinician inserted the drain with USS evidence only and did not have access to the CT scan at the time of insertion to reflect clinical practice. A scoring system was developed for each individual radiological modality and used to compare imaging characteristics of the pleural fluid collection and underlying parenchyma and to assess the utility of USS and CT to predict length of stay after the intervention.

Results: Of the 60 subjects recruited, 46 had USS images available for review, 36 had a CT scan which met the inclusion criteria and 31 had all three radiological measurements (CT, USS and CXR) available for analysis. There was substantial interobserver agreement for USS grades $(\kappa=0.709)$ and moderate agreement for total CT scores $(\kappa=0.520)$. There were weak correlations between USS grade and total CT score as well as CT loculation and density scores. Of the 25 CXRs showing simple opacification of the underlying parenchyma only, CT demonstrated simple consolidation $(n=14)$, necrotising pneumonia $(n=7)$, cavitary necrosis $(n=3)$ and pneumatoceles $(n=1)$. No abnormality was detected on CT scanning which directly altered clinical management. Neither the USS score nor the CT score, nor a combination of the two, were able to predict length of hospital stay.

Conclusions: CT scanning detects more parenchymal abnormalities than chest radiography. However, the additional information does not alter management and is unable to predict clinical outcome. This suggests that there is no role for the routine use of CT scanning in children if treated with urokinase and percutaneous chest drain. The omission of routine CT scanning in empyema will reduce the exposure of children to unnecessary radiation and reduce costs.

Trial registration number: The trial is fully registered with clinicaltrials.gov (ID: NCT00144950).

Although relatively uncommon, the incidence of empyema in children is increasing in many countries. ${ }^{1-4}$ The British Thoracic Society (BTS) guidelines on the management of empyema in children highlighted the lack of grade A evidence to inform best management. ${ }^{5}$ Since its publication in 2005, this has been increasingly addressed by the publication of prospective randomised controlled studies. ${ }^{67}$ The BTS guidelines further highlighted the lack of evidence to inform imaging modalities to assist in management. The consensus was that a posterioanterior (or anteroposterior) chest radiograph (CXR) together with a pleural ultrasound scan (USS) should be performed. The expert opinion (level D evidence) was that chest computed tomography (CT) should not be performed routinely but that there may be a role in atypical empyema presentations.

If a parapneumonic effusion contains pus it is called an empyema. The American Thoracic Society (ATS) divided the empyema process into three stages: (1) exudative, in which the pleural fluid has a low cell content; (2) fibrinopurulent, in which frank pus is present and fibrin formation begins to cover the pleura with formation of loculations; and (3) organising phase, in which there is thick peel formation by fibroblasts and the pleural space is characterised by "very thick exudates with heavy sediment". " USS has been used to stage this process in children by demonstrating the presence of septations and to guide management; ${ }^{9}$ however, the role of routine CT scanning in guiding management in children with empyema is not known. A major limitation of the radiological data available from adult studies is that they are not applicable to the paediatric population. ${ }^{10}$ The disease rarely causes death in children, who are generally healthy before the onset of infection compared with adult empyema which has an estimated mortality of $20 \% .{ }^{11}$

Management options include the insertion of a drain with or without fibrinolytics, ${ }^{6}{ }^{12}$ limited open decortication ${ }^{9}$ or video-assisted thoracoscopic surgery (VATS). ${ }^{13}{ }^{14}$ In most cases where a surgical approach is taken, a CT scan is performed preoperatively. This is particularly true in VATS where the surgeon requires a "road map" to provide greater anatomical delineation and better definition of the underlying lung parenchyma to ensure that the placement of the instruments does not cause a bronchopleural fistula. In those centres where children are managed with urokinase and percutaneous chest drain insertion, a CT scan is not routinely performed. There is no consensus on radiological investigations in childhood empyema and practice varies between centres.

The aims of this study were to develop a radiological scoring system for childhood empyema and to assess the utility of routine CT chest scanning in paediatric empyema. 


\section{METHODS}

This study formed part of a previously published prospective randomised study comparing VATS with percutaneous drain insertion and urokinase. ${ }^{6}$ Subjects were recruited from children aged $<16$ years referred to our institution, a tertiary paediatric respiratory centre, for further management of complicated parapneumonic effusion over a 3-year period. Preoperative CXR, pleural USS and CT scanning were performed routinely in all patients, irrespective of the treatment group to which they were randomised. However, in order to reflect local standard clinical practice, the CT scans in the VATS group were made available to the surgeons. In the urokinase group the radiologist and paediatrician in charge were aware of the CT image findings; however, at the time of insertion, the clinician inserting the drain did not have the CT information available unless concerns had been raised by the senior clinician or radiologist. The primary clinical outcome measure used for this radiological study was length of stay in hospital following the intervention.

The comparisons between CT and both USS and CXR were twofold: (1) we compared the imaging characteristics of the pleural fluid collection itself as defined by USS and CT; and (2) we compared the evaluation of the underlying pulmonary parenchyma as demonstrated on CXR and CT scans as described below.

\section{Pleural collections}

Ultrasound (USS) evaluation

Patients were referred for a pleural USS examination before treatment (see online supplement for detailed explanation of methodology). The images were subsequently reviewed by two paediatric radiologists (AC, $\mathrm{CMO}$ ) blinded to patient data. The effusions were graded according to their internal echostructure using a system based on that described by Kearney et a ${ }^{15}$ but with the addition of an extra grade for highly septated effusions with significant solid components (grade 1, anechoic; grade 2, echoic fluid without septation; grade 3, septated effusion; grade 4 , septations with solid appearing components comprising more than one-third of the effusion; see fig 1). Scores from both radiologists were used for analysis.

\section{CT evaluation}

CT scans were performed before treatment in all patients. Scans were excluded from analysis if they were performed without intravenous iodinated contrast material as intravenous contrast allows visualisation of pleural inflammation which is not normally possible with a non-contrast enhanced scan. We also excluded scans done at a local hospital prior to admission and transferred with a hard copy or performed following chest drain insertion (see online supplement for detailed explanation of CT methodology). Effusions were graded by fluid density, pleural enhancement, subcostal tissue thickening and loculation/effusion shape (table 1).

Effusions were graded as follows: effusion density was taken as the mean of the CT density in Hounsfield units (HU) recorded from three regions of interest drawn in the centre of the thickest portion of the effusion at three contiguous levels measured by one observer (AC): a density of $0-20$ scored 0 and a density of $>20$ scored 1 . A cut-off point of $20 \mathrm{HU}$ was chosen as this is generally the accepted upper limit for the density of water-based lesions, for example, in hepatic and renal cysts. The remaining evaluations were performed independently by two paediatric radiologists (AC and $\mathrm{CMO}$ ).

Parietal pleural enhancement recorded a score of 1 , and a score of 2 was given for thick pleural enhancement $(>2 \mathrm{~mm}$ ). Thickening of the subcostal tissues was scored similarly.

We devised a scoring system to reflect the degree of loculation of the effusion through the assessment of effusion shape: an effusion whose visceral surface paralleled the chest wall scored 0 , an effusion with convexity or lobulation of the visceral surface with pleura/pleural fluid thickness between the lobulations of
Figure 1 Ultrasound staging of empyema. (A) Grade 1 effusion showing anechoic fluid. (B) Grade 2 effusion showing echoic fluid without septations. (C) Grade 3 effusion showing multiple thick septations. (D) Grade 4 effusion showing multiple septations with solid appearing components comprising more than one-third of the collection.
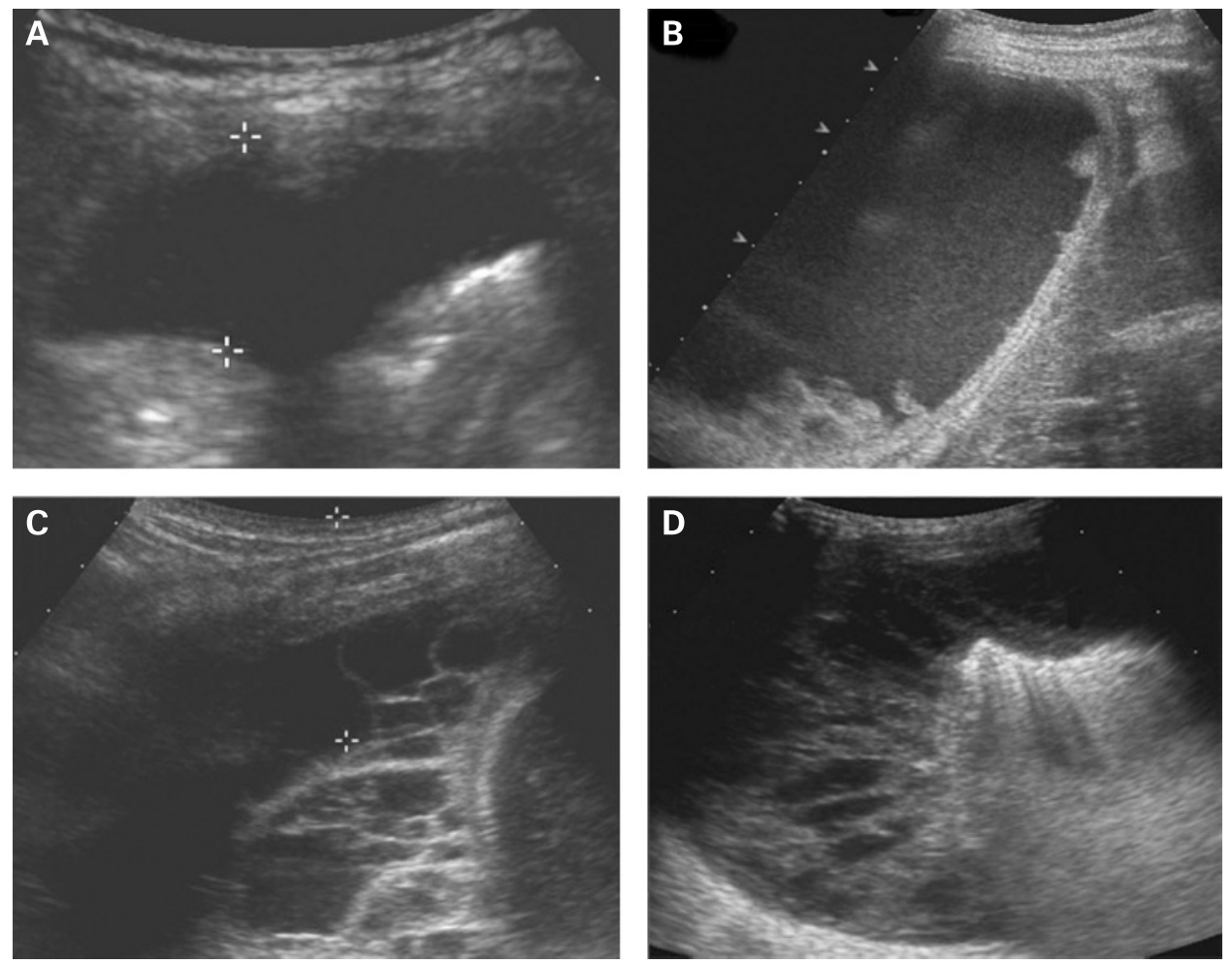
Table 1 CT scoring system for parapneumonic effusions (maximum total score $=8$ )

\begin{tabular}{|c|c|c|c|}
\hline Fluid density & Pleural enhancement & $\begin{array}{l}\text { Subcostal tissue } \\
\text { thickening }\end{array}$ & Loculation/effusion shape \\
\hline \multirow[t]{2}{*}{$\begin{array}{l}\text { Mean density }<20 \mathrm{HU} \\
\text { (Score } 0 \text { ) }\end{array}$} & $\begin{array}{l}\text { Absent } \\
\text { (Score 0) }\end{array}$ & $\begin{array}{l}\text { Absent } \\
\text { (Score 0) }\end{array}$ & $\begin{array}{l}\text { Simple: Concave medially, parallels chest wall } \\
\text { (Score 0) }\end{array}$ \\
\hline & $\begin{array}{l}\text { Present, }<2 \mathrm{~mm} \text { thick } \\
\text { (Score 1) }\end{array}$ & $\begin{array}{l}\text { Present, }<2 \mathrm{~mm} \text { thick } \\
\text { (Score 1) }\end{array}$ & $\begin{array}{l}\text { Loculated: convex medially or lobulated with } \\
\text { separation of lobulations by }>10 \mathrm{~mm} \text { of fluid/ } \\
\text { thickening } \\
\text { (Score 1) }\end{array}$ \\
\hline \multirow[t]{2}{*}{$\begin{array}{l}\text { Mean density }>20 \mathrm{HU} \\
\text { (Score 1) }\end{array}$} & $\begin{array}{l}\text { Present, }>2 \mathrm{~mm} \text { thick } \\
\text { (Score 2) }\end{array}$ & $\begin{array}{l}\text { Present, }>2 \mathrm{~mm} \text { thick } \\
\text { (Score 2) }\end{array}$ & $\begin{array}{l}\text { Incomplete multiloculation: lobulations } \\
\text { separated by }<10 \mathrm{~mm} \text { of pleural thickness } \\
\text { (Score 2) }\end{array}$ \\
\hline & & & $\begin{array}{l}\text { Complete multiloculation: pockets of fluid } \\
\text { separated by normal intervening pleura } \\
\text { (Score 3) }\end{array}$ \\
\hline
\end{tabular}

HU, Hounsfield units.

$>10 \mathrm{~mm}$ scored 1 , and an effusion whose visceral surface showed lobulations separated by $<10 \mathrm{~mm}$ of pleural fluid or thickening scored 2 (fig 2). If components of the pleural collection were separated by normal pleura, the effusion scored 3 . The individual scores were summated to give one total score and scores from both observers were used for analysis.

\section{Pulmonary parenchyma}

Chest radiograph (CXR) evaluation

Posterioanterior CXRs were performed preoperatively in all subjects. The immediate preoperative CXRs were included in the study even if performed at local hospitals before referral to our tertiary unit. Parenchymal changes were classified as simple opacification (including collapse and consolidation with or without air bronchograms) and opacification with cavitation. The CXRs were blindly evaluated by two independent radiologists (AC, CMO) and one paediatric pulmonologist (SS), reporting in consensus, reaching a conclusion by mutual discussion.

\section{CT evaluation}

Pulmonary parenchymal changes were classified in consensus by mutual discussion by two radiologists (AC, CMO) based on the definitions described by Donnelly et al: ${ }^{16}$

- Simple collapse or consolidation: homogeneous opacification and enhancement present with or without air bronchograms or with fluid-filled airways.

- Pneumatoceles: tubular or cystic areas of air density with thin or imperceptible walls, with no evidence of necrotising pneumonia.

- Necrotising pneumonia: ill-defined areas of poorly enhancing lung comprising more than half of consolidated lung.
- Cavitary necrosis: necrotising pneumonia containing irregular areas of air density.

- Pulmonary abscess: well-defined area of intrapulmonary fluid density or cavity with air fluid level, with thick (>2 mm) enhancing wall.

\section{Statistical analysis}

Interobserver reliability for the CT and USS scores between the two observers was assessed using Cohen's kappa ( $\kappa$ ) with linear weighting. Correlation between USS grade and both the total CT score and the individual component scores were calculated using Kendall's rank correlation. The utility of CT and USS scores to predict the number of days in hospital after the intervention was assessed using multiple linear regression analysis adjusted for intervention (VATS vs urokinase).

\section{RESULTS}

Of the 60 subjects randomised to either VATS or urokinase, 46 had USS images available for review. Thirty-six patients had a CT scan which met the inclusion criteria. Exclusions were as follows: CT performed at local hospital $(n=14)$; no contrast enhancement due to failure of intravenous access $(n=2)$; performed with drain in situ $(n=3)$; unavailable for review $(n=5)$. Thirty-one patients had all three radiological measurements (CT, USS and CXR) available for analysis.

\section{Pleural collection evaluation}

There was substantial interobserver agreement for USS grades $(\kappa=0.709)$ and moderate agreement for total CT scores ( $\kappa=0.520$, tables 2 and 3 ). Agreement between the two observers for CT scores was strongest for the subcostal thickening $(\kappa=0.568)$ and pleural enhancement scores
Figure 2 CT shape score. (A) Shape score of 1 , effusion showing lobulations with intervening pleural space of $>10 \mathrm{~mm}$. (B) Shape score of 2 showing lobulations (arrows) separated by narrow bridge of pleural thickening.
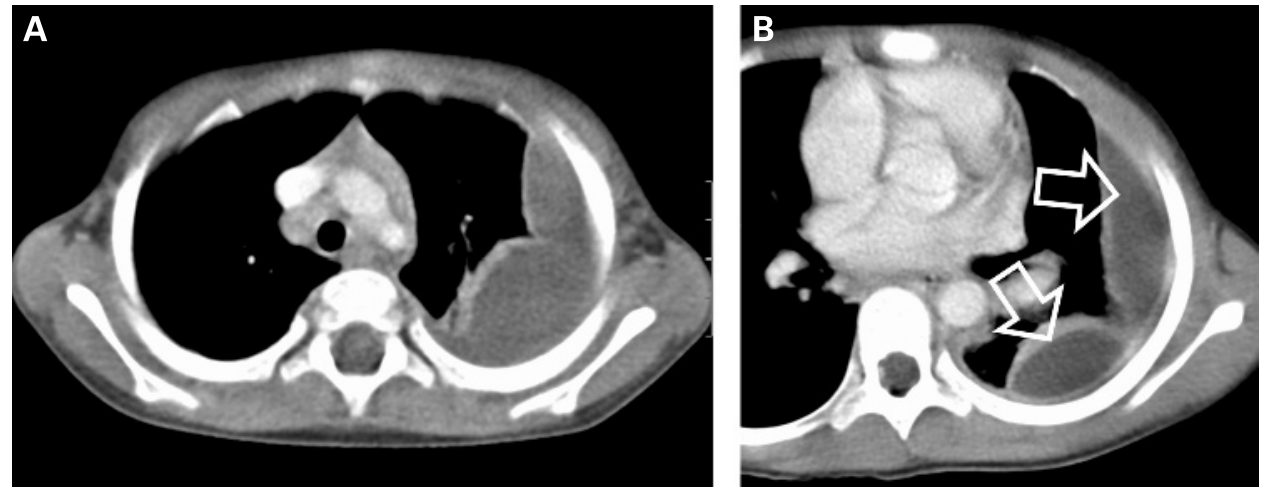
Table 2 Frequency of ultrasound grades by observers ( $\kappa=0.709$ )

\begin{tabular}{lrrrrr}
\hline & \multicolumn{5}{c}{ Observer $\mathbf{2}$ ultrasound grades } \\
\cline { 2 - 6 } & $\mathbf{1}$ & $\mathbf{2}$ & $\mathbf{3}$ & $\mathbf{4}$ & Total \\
\hline Observer 1 ultrasound grades & & & & & \\
1 & 1 & 0 & 0 & 0 & 1 \\
2 & 0 & 6 & 2 & 0 & 8 \\
3 & 0 & 1 & 10 & 2 & 13 \\
4 & 0 & 1 & 1 & 7 & 9 \\
Total & 1 & 8 & 13 & 9 & 31 \\
\hline
\end{tabular}

$(\kappa=0.459)$. Five of 31 effusions showed increased density (score 1 on density score). The majority of effusions showed pleural enhancement (scores 1 and 2:20/31 for observer 1 and 25/31 for observer 2). Subcostal tissue thickening was present in a minority (scores 1 and 2: 15/31 for observer 1 and 10/31 for observer 2). The majority of effusions showed some degree of loculation (internal convexity and incomplete multiloculation, scores 1-2, 23/31 for observer 1 and 24/31 for observer 2). However, we did not identify any cases of completely multiloculated collections (score 3 on loculation/shape score) where locules were completely separated from each other by normal intervening pleura.

The mean CT score increased across the USS grades for pooled observations (data not shown) with an incremental increase in mean shape, pleural thickening and density score from USS grades 2-4 (only one patient had a USS grade of 1 for each observer). There were weak correlations between USS grade and total CT score as well as the CT loculation score and CT density score (table 4). Despite the positive and statistically significant correlation between USS and CT score, the strength of the relationship is minimal as demonstrated by the low correlation coefficients.

\section{Pulmonary parenchyma}

Twenty-five of the CXRs showed simple opacification of the underlying parenchyma only. Of these, 14 had simple consolidation only, in one case pneumatoceles were present, in seven cases necrotising pneumonia was present and in three cavitary necrosis was present (table 5). Of the six cases with cavitation on the CXR, four showed cavitary necrosis on the CT scan, one showed pneumatoceles and one showed simple consolidation only.

No abnormality was detected on CT scanning which directly altered management. In particular, we did not identify any cases of pulmonary abscess although, in several cases, areas of cavitary necrosis were very well defined but lacked an enhancing wall and therefore did not meet our criteria (fig 3).

\section{Outcome prediction}

In the primary study there was no statistically significant difference in length of hospital stay following intervention between the two treatment groups: median difference 0 ( $95 \%$ CI -1 to 2$),(p=0.311) .{ }^{6}$ The small sample size in this study precluded comprehensive evaluation of the predictive abilities of the radiographic modalities, although basic multivariable regression adjusted for treatment showed that neither the USS score (geometric slope 1.06 (95\% CI 0.72 to 1.59 ), $\mathrm{p}=0.744)$ nor the CT score (geometric slope $1.09(95 \% \mathrm{CI}$ 0.96 to 1.23), $p=0.182$ ) nor a combination of the two (geometric slope 1.08 (95\% CI 0.97 to 1.19 ), $\mathrm{p}=0.151$ ) were able to predict length of hospital stay. Patients with CT findings
Table 3 Frequency of total CT scores by observer $(\kappa=0.520)$

\begin{tabular}{lllllllll}
\hline & \multicolumn{1}{c}{ Observer $\mathbf{2}$ total CT scores } \\
\cline { 2 - 8 } & $\mathbf{0}$ & $\mathbf{1}$ & $\mathbf{2}$ & $\mathbf{3}$ & $\mathbf{4}$ & $\mathbf{5}$ & $\mathbf{6}$ & Total \\
\hline $\begin{array}{l}\text { Observer 1 total CT } \\
\text { scores }\end{array}$ & & & & & & & & \\
0 & 1 & 2 & 1 & 0 & 0 & 0 & 0 & 4 \\
1 & 1 & 1 & 3 & 0 & 0 & 0 & 0 & 5 \\
2 & 1 & 1 & 1 & 2 & 0 & 0 & 0 & 5 \\
3 & 0 & 0 & 3 & 2 & 1 & 0 & 0 & 6 \\
4 & 0 & 1 & 0 & 1 & 1 & 2 & 0 & 5 \\
5 & 0 & 0 & 0 & 0 & 2 & 0 & 1 & 3 \\
6 & 0 & 0 & 0 & 0 & 1 & 0 & 1 & 2 \\
Total & 3 & 6 & 10 & 8 & 9 & 7 & 8 & 31 \\
\hline
\end{tabular}

other than simple consolidation or pneumatoceles did not have a different outcome. Patients in the VATS arm with simple consolidation $(n=7)$ had a median stay of 5 days (range 4-16) and those with complicated parenchymal disease $(n=7)$ a median stay of 5 days (range 4-12). Patients in the urokinase arm and simple consolidation $(n=10)$ had a median stay of 5 days (range 5-9) while those with complicated parenchymal disease $(n=7)$ had a median stay of 8 days (range 5-16).

\section{DISCUSSION}

We have developed a novel radiological scoring system for children with empyema and demonstrated that CT scans detect more parenchymal changes than CXRs and that no additional abnormality was detected which directly changed clinical management. Furthermore, neither modality alone or in combination was able to predict the clinical outcome and therefore appear to be poor markers of disease severity, albeit these analyses are based on small numbers. These findings suggest no role for routine CT scanning in the management of children with empyema if managed with percutaneous drainage and urokinase.

USS is a central investigation in the management of paediatric empyema, offering several key strengths: it is portable, non-invasive, does not use ionising radiation and provides a dynamic assessment of the chest. It is cheap and relatively easy to perform and is able to differentiate pleural fluid from consolidation, estimate the effusion size and guide chest drain placement. It is also able to demonstrate the presence of fibrinous septations within pleural collections. ${ }^{17-19}$

CT scanning has several potential strengths as it is able to provide an examination of the entire thorax without being limited by the presence of air or bone compared with USS. It is also able to provide an accurate "road map" of the pleural space for an operating surgeon. CT can provide an accurate assessment of the pulmonary parenchyma beneath an effusion, the assessment of which is often limited on chest radiography by the presence of pleural fluid. However, CT has many disadvantages: it is unable to detect the presence of fibrinous septations, which are usually too thin and of insufficient density to identify, ${ }^{17}{ }^{19}$ and requires sedation or anaesthesia in an uncooperative child. The principal weakness of CT, however, is its use of ionising radiation. At our institution the CT dose is estimated to be approximately 115 times that of a $\mathrm{CXR},{ }^{20}$ and this may be considerably higher in units not using paediatric dose optimisation. Radiation dose reduction is of particular importance in children as they are more susceptible to the risks of radiation and have a substantially increased lifetime cancer risk compared with the older population following a single CT 
Table 4 Correlation coefficients (Kendall's tau) between ultrasound (USS) grade and CT scores from pooled observations

\begin{tabular}{lll}
\hline Correlation & Kendall's tau (95\% CI) & p Value \\
\hline USS grade vs CT score & $0.308(0.118$ to 0.512$)$ & 0.0035 \\
USS grade vs CT density score & $0.271(0.0351$ to 0.458$)$ & 0.0246 \\
$\begin{array}{l}\text { USS grade vs CT pleural enhancement } \\
\text { score }\end{array}$ & $0.208(-0.0218$ to 0.448$)$ & 0.0716 \\
$\begin{array}{l}\text { USS grade vs subcostal thickening } \\
\text { score }\end{array}$ & $0.188(-0.0475$ to 0.402$)$ & 0.1036 \\
USS grade vs CT shape score & $0.367(0.132$ to 0.563$)$ & 0.0014 \\
\hline
\end{tabular}

scan. ${ }^{21}$ A CT examination in a child can thus only be justified if the result is likely to impact on clinical management. This study has shown that, although CT scans were able to detect more detailed parenchymal findings than CXR in our population, no CT scan detected an unexpected abnormality which directly altered clinical management-for example, the detection of an unexpected lung abscess requiring prolonged antibiotics ${ }^{22}$ or drainage. ${ }^{23}$

There are no well validated scoring systems for the severity of empyema measured by CT. Donnelly and Klosterman ${ }^{24}$ used a simple 4-point system based on pleural and subcostal fat thickening along with changes in the chest wall. They did not assess the density of pleural fluid or loculation/effusion shape and did not perform independent observations with this system (and hence no measure of interobserver variation was given). This scoring system was unable to distinguish between empyema and transudative effusions. Kearney et $a l^{15}$ assessed severity of effusions by size, loculation, pleural enhancement, pleural thickening and pleural septation, but did not combine these features into an overall score. In the absence of a validated scoring system, we devised a novel system for scoring CT findings in empyema, based on previously described features of pleural thickening and thickening of subcostal fat, along with assessments of effusion density and loculation. We found only moderate interobserver agreement for this scoring system. This partly reflects a difficulty with all CT scoring systems of reducing a complex three-dimensional dataset to a single score. Some of the components of the score-particularly the shape score-are quite subjective and hence prone to interobserver variability. We believe the relatively poor level of agreement achieved further highlights the difficulty of using CT as a measure of disease severity in empyema. Furthermore, CT and USS findings do not appear to show a strong relationship, reflecting the very different basis and strengths of these two modalities.

Neither the USS grade nor the CT score, nor a combination of the two, was able to predict outcome. There are very few studies which have used radiology to attempt to predict outcome. In a study of 50 children with empyema who underwent either primary or secondary thoracoscopy, the clinical outcome was not predicted by USS when assessed for echogenicity, thickness of the effusion and presence of septations. ${ }^{25}$ Similarly, in a prospective randomised study of intrapleural urokinase to treat empyema in children, USS findings were not related to outcome. ${ }^{12}$ Kearney et al compared USS and CT scanning in a study of 50 adult patients with parapneumonic effusions. Neither the USS grade nor CT findings of effusion size, pleural thickening or extrapleural fat thickening were able to reliably stage empyema or predict failure of tube drainage. ${ }^{15}$ We have previously published data demonstrating no difference in clinical outcome between VATS or treatment with urokinase. However, the VATS group was
Table 5 Parenchymal changes detected on CT scan and chest radiograph (CXR)

\begin{tabular}{lrlc}
\hline & \multicolumn{2}{l}{ CXR findings } \\
\cline { 2 - 4 } CT findings & Simple & Cavitation & Total \\
\hline Simple & 14 & 1 & 15 \\
Pneumatoceles & 1 & 1 & 2 \\
Necrotising pneumonia & 7 & 0 & 7 \\
Cavitary necrosis & 3 & 4 & 7 \\
Abscess & 0 & 0 & 0 \\
Total & 25 & 6 & 31 \\
\hline
\end{tabular}

significantly more expensive, particularly with the addition of a CT scan. ${ }^{6}$ Our study data do not support the use of USS, CT or a combination as a means of predicting the outcome from empyema treatment in children, further supporting the view that the role of routine CT scanning in childhood empyema is questionable.

In our analysis of parenchymal findings, CT demonstrated potentially important findings of necrotising pneumonia or cavitary necrosis in $10 / 25$ patients with apparently simple collapse or consolidation on the CXR. The finding that CT scanning detects more parenchymal changes than chest radiography supports previous published evidence. ${ }^{16} 1926$ Empyema appears to be a common accompaniment to cavitary necrosis. ${ }^{16} 27$ Importantly, in our study, patients with cavitary necrosis or necrotising pneumonia on CT scanning in addition to empyema showed no difference in outcome from those with simple consolidation, although the numbers involved are small.

This study is subject to several limitations. We recognise that our sample size is small. The initial clinical study was powered to detect a difference of 2 days in the primary outcome measure (number of days in hospital post-intervention) between the two treatment groups and was not powered to detect post hoc differences between radiological modalities. Furthermore, only 31 of the 60 patients recruited had a complete set of valid imaging studies available for review in this study. Of particular note is that 14 patients had CT scans carried out at the local hospital before referral, highlighting the low threshold physicians have for performing CT scans on children with empyema.

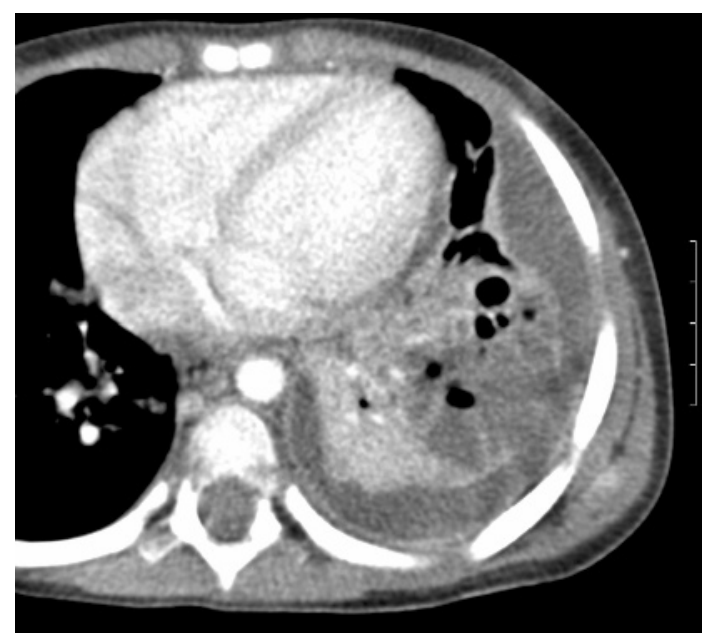

Figure 3 Well-defined cavitary necrosis. CT image showing welldefined area of non-enhancing lung with cavitation adjacent to empyema. As this area lacks an enhancing wall, this could not be defined as an abscess. 
Another limitation is the retrospective nature of our radiological analysis, particularly for USS. USS is a real-time and dynamic examination that can never be fully represented in stored or printed images. The accuracy of our grading is thus open to question. The high level of interobserver variability obtained from independent observations does at least partly validate our methodology. Owing to the retrospective nature of radiological analysis, we were not able to analyse pulmonary parenchymal changes or effusion shape on USS as these require a real-time assessment.

A further potential limitation is that we did not directly assess the intervention of CT scanning by randomising subjects to receive CT or not, which we believe would have been unethical. The primary aim of the study was to compare two clinical interventions and the study was designed to reflect standard clinical practice where surgeons routinely request a CT scan before performing VATS, while percutaneous drains are inserted under USS guidance without the need of a CT scan. Nevertheless, we believe that the study design reflects a pragmatic approach and represents the first study to examine the role of routine CT scanning in childhood empyema providing an evidence base for management of this disease.

In conclusion, using the novel radiological scoring system, we identified only weak correlation between CT and USS findings in paediatric pleural empyema. Neither CT nor USS provides a reliable means of predicting outcome. Although CT detects additional parenchymal findings to those identifiable on CXR, these do not influence management in the setting of empyema. This study suggests no role for the routine use of CT in the management of pleural empyema in children if treated with percutaneous drainage and urokinase. This approach will reduce the exposure of children to unnecessary radiation and reduce costs.

Acknowledgements: The authors thank Dr Robert Dinwiddie, Dr Colin Wallis, Dr Paul Aurora (respiratory paediatricians), Dr Gordon Cohen, Dr Carin van Doorn, Professor Martin Elliott (paediatric thoracic surgeons) and Mr John Cairns.

Funding: Research at the Institute of Child Health and Great Ormond Street Hospital for Children NHS Trust benefits from Research and Development funding received from the NHS Executive.

Competing interests: $\mathrm{AJ}$ is in receipt of an unrestricted grant from GlaxoSmithKline (Belgium) for a study on the epidemiology of childhood empyema in Australia and New Zealand.

Ethics approval: The project was approved by the local ethics committee.

\section{REFERENCES}

1. Rees JH, Spencer DA, Parikh D, et al. Increase in incidence of childhood empyema in West Midlands, UK. Lancet 1997;349:402.

2. Playfor SD, Smyth AR, Stewart RJ. Increase in incidence of childhood empyema. Thorax 1997;52:932.

3. Byington CL, Spencer LY, Johnson TA, et al. An epidemiological investigation of a sustained high rate of pediatric parapneumonic empyema: risk factors and microbiological associations. Clin Infect Dis 2002;34:434-40.

4. Spencer DA, lqbal SM, Hasan A, et al. Empyema thoracis is still increasing in UK children. BMJ 2006; 332:1333.

5. Balfour-Lynn IM, Abrahamson E, Cohen G, et al. BTS Guidelines for the management of pleural infection in children. Thorax 2005;60(Suppl 1):i1-21.

6. Sonnappa S, Cohen G, Owens CM, et al. Comparison of urokinase and videoassisted thoracoscopic surgery for treatment of childhood empyema. Am J Respir Crit Care Med 2006;174:221-7.

7. Kurt BA, Winterhalter KM, Connors RH, et al. Therapy of parapneumonic effusions in children: video-assisted thoracoscopic surgery versus conventional thoracostomy drainage. Pediatrics 2006;118:e547-53.

8. American Thoracic Society. Management of nontuberculous empyema. Am Rev Respir Dis 1962:85:935-6.

9. Carey JA, Hamilton JR, Spencer DA, et al. Empyema thoracis: a role for open thoracotomy and decortication. Arch Dis Child 1998:79:510-3.

10. Jaffe $\mathbf{A}$, Balfour-Lynn IM. Management of empyema in children. Pediatr Pulmonol 2005;40:148-56.

11. Ferguson AD, Prescott RJ, Selkon JB, et al. The clinical course and management of thoracic empyema. O J Med 1996;89:285-9.

12. Thomson $\mathbf{A H}$, Hull J, Kumar MR, et al. Randomised trial of intrapleural urokinase in the treatment of childhood empyema. Thorax 2002;57:343-7.

13. Cohen G, Hjortdal V, Ricci MJ, et al. Primary thoracoscopic treatment of empyema in children. J Thorac Cardiovasc Surg 2003;125:79-84.

14. Jaffe A, Cohen G. Thoracic empyema. Arch Dis Child 2003:88:839-41.

15. Kearney SE, Davies CW, Davies RJ, et al. Computed tomography and ultrasound in parapneumonic effusions and empyema. Clin Radiol 2000;55:542-7.

16. Donnelly LF, Klosterman LA. Cavitary necrosis complicating pneumonia in children: sequential findings on chest radiography. Am J Roentgenol 1998;171:253-6.

17. Patel MC, Flower CD. Radiology in the management of pleural disease. Eur Radiol 1997:7:1454-62.

18. King S, Thomson A. Radiological perspectives in empyema. Br Med Bull 2002;61:203-14.

19. Evans AL, Gleeson FV. Radiology in pleural disease: state of the art. Respirology 2004:9:300-12.

20. Papaioannou G, Young C, Owens CM. Multidetector row CT for imaging the paediatric tracheobronchial tree. Pediatr Radiol 2007;37:515-29.

21. Brenner DJ, Hall EJ. Computed tomography: an increasing source of radiation exposure. N Engl J Med 2007;357:2277-84.

22. Chan PC, Huang LM, Wu PS, et al. Clinical management and outcome of childhood lung abscess: a 16-year experience. J Microbiol Immunol Infect 2005:38:183-8.

23. Coren ME, Ng M, Rubens $\mathrm{M}$, et al. The value of ultrafast computed tomography in the investigation of pediatric chest disease. Pediatr Pulmonol 1998;26:389-95.

24. Donnelly LF, Klosterman LA. CT appearance of parapneumonic effusions in children: findings are not specific for empyema. Am J Roentgenol 1997;169:179-82.

25. Kalfa N, Allal $\mathrm{H}$, Lopez $\mathbf{M}$, et al. Thoracoscopy in pediatric pleural empyema: a prospective study of prognostic factors. J Pediatr Surg 2006;41:1732-7.

26. Donnelly LF, Klosterman LA. The yield of CT of children who have complicated pneumonia and noncontributory chest radiography. Am J Roentgenol 1998;170:1627-31

27. Ramphul N, Eastham KM, Freeman R, et al. Cavitatory lung disease complicating empyema in children. Pediatr Pulmonol 2006:41:750-3. 